\title{
Association of pregnancy outcomes in women with type 2 diabetes treated with metformin versus insulin when becoming pregnant
}

Shu-Fu Lin ${ }^{1,2,3}$, Shang-Hung Chang 3,4,5, Chang-Fu Kuo ${ }^{3,7,8}$, Wan-Ting Lin ${ }^{5}$, Meng-Jiun Chiou ${ }^{8}$ and Yu-Tung Huang ${ }^{5^{*}}$ (D)

\begin{abstract}
Background: Metformin use in pregnancy is controversial because metformin crosses the placenta and the safety on the fetus has not been well-established. This retrospective study aimed to compare pregnancy outcomes in women with preexisting type 2 diabetes receiving metformin or standard insulin treatment.

Methods: The cohort of this population-based study includes women of age 20-44 years with preexisting type 2 diabetes and singleton pregnancies in Taiwan between 2003 and 2014. Subjects were classified into three mutually exclusive groups according to glucose-lowering treatments received before and after becoming pregnant: insulin group, switching group (metformin to insulin), and metformin group. A generalized estimating equation model adjusted for patient age, duration of type 2 diabetes, hypertension, hyperlipidemia, retinopathy, and aspirin use was used to estimate the adjusted odds ratio (aOR) and 95\% confidence interval (Cl) of adverse pregnancy outcomes.

Results: A total of 1166 pregnancies were identified in the insulin group $(n=222)$, the switching group $(n=318)$ and the metformin group $(n=626)$. The insulin group and the switching group had similar pregnancy outcomes for both the mother and fetus, including risk of primary cesarean section, pregnancy-related hypertension, preeclampsia, preterm birth ( $<37$ weeks), very preterm birth $(<32$ weeks), low birth weight $(<2500 \mathrm{~g})$, high birth weight (> $4000 \mathrm{~g}$ ), large for gestational age, and congenital malformations. The metformin group had a lower risk of primary cesarean section $(\mathrm{aOR}=0.57 ; 95 \% \mathrm{Cl}, 0.40-0.82)$ and congenital malformations $(\mathrm{aOR}, 0.51 ; 95 \% \mathrm{Cl} ; 0.27-0.94)$ and similar risk for the other outcomes as compared with the insulin group.
\end{abstract}

Conclusions: Metformin therapy was not associated with increased adverse pregnancy outcomes in women with type 2 diabetes as compared with standard insulin therapy.

Keywords: Pregnancy outcome, Metformin, Insulin

\footnotetext{
* Correspondence: anton.huang@gmail.com

${ }^{5}$ Center for Big Data Analytics and Statistics, Chang Gung Memorial Hospital, Linkou, No.15, Wunhua 1st Rd., Gueishan Dist, Taoyuan City 333, Taiwan

Full list of author information is available at the end of the article
}

(c) The Author(s). 2020 Open Access This article is licensed under a Creative Commons Attribution 4.0 International License, which permits use, sharing, adaptation, distribution and reproduction in any medium or format, as long as you give appropriate credit to the original author(s) and the source, provide a link to the Creative Commons licence, and indicate if changes were made. The images or other third party material in this article are included in the article's Creative Commons. licence, unless indicated otherwise in a credit line to the material. If material is not included in the article's Creative Commons licence and your intended use is not permitted by statutory regulation or exceeds the permitted use, you will need to obtain permission directly from the copyright holder. To view a copy of this licence, visit http://creativecommons.org/licenses/by/4.0/ The Creative Commons Public Domain Dedication waiver (http://creativecommons.org/publicdomain/zero/1.0/) applies to the data made available in this article, unless otherwise stated in a credit line to the data. 


\section{Background}

Type 2 diabetes in pregnancy is a growing concern as the prevalence of type 2 diabetes in younger women is increasing and type 2 diabetes in pregnancy confers an increased risk of adverse pregnancy outcomes [1, 2]. Achievement of optimal glucose control before and during pregnancy is pivotal in order to minimize the occurrence of pregnancy complications [3]. Lifestyle modifications, including medical nutrition therapy and increased physical activity, are important in the management of type 2 diabetes; however, lifestyle interventions are usually insufficient to achieve glycemic targets in many patients and pharmacotherapy is frequently required $[3,4]$.

Insulin is the standard drug for the management of type 2 diabetes in pregnancy because it is effective for glycemic control and does not cross the placenta [3]. However, there are some barriers to insulin initiation, including injection phobia [5]. Metformin is an oral glucose-lowering agent that does not increase the risk of hypoglycemia and is the first-line pharmacological treatment for patients with type 2 diabetes [6-8]. The safety of metformin use in pregnancy has been reported in women with polycystic ovary syndrome (PCOS), gestational diabetes mellitus (GDM) and obesity [9-14]. However, the use of metformin during pregnancy remains controversial because metformin freely crosses the placenta and reaches concentrations in the fetus equivalent to those of the mother $[15,16]$. USA guidelines suggest that metformin should be discontinued and insulin should be started as soon as possible when pregnancy occurs in patients with type 2 diabetes [3]. Current Australian guideline, however, is less restrictive [17].

In this study, we used national population cohort databases in Taiwan to compare pregnancy outcomes in women who received metformin or standard insulin treatment during pregnancy.

\section{Methods}

\section{Data sources}

The Taiwan National Health Insurance (NHI) is a single-payer system established in 1995 that provides universal medical care coverage to over $99 \%$ of the Taiwan residents (approximately 23.5 million people), including all citizens and foreigners living in Taiwan for more than 6 months [18]. This study analyzed data from the Taiwan NHI database, which was initiated in 1997 by the NHI Administration to facilitate research on health care. This database contains comprehensive information regarding patient demographic profiles (date of birth, sex, place of residence, and income level), detailed clinical information (diagnoses associated with inpatient and outpatient care and procedures), prescribed medications and surgeries, and fees incurred. Personal identification has been anonymized but kept consistent between NHI databases and other government-held datasets to facilitate accurate linkage [19].

The Taiwan Birth Registry dataset was also included in this study for the analysis of pregnancy outcomes. This data contains detailed birth characteristics of the mother and fetus, including method of delivery, gestational age, birth weight, Apgar score, congenital malformations, and stillbirth. In Taiwan, obstetricians have been required to report these data for the fetus at $\geq 20$ weeks' gestation to the Ministry of Health and Welfare since 1995. The Taiwan Birth Registry dataset has been shown to have high validity [20]. Both NHI and Birth Registry databases were approved to use for this study by the Health and Welfare Data Science Center, Ministry of Health and Welfare, Taiwan.

\section{Study cohort}

Data in the Taiwan NHI claim dataset and Birth Registry dataset between January 1, 2003 and December 31, 2014 were retrospectively analyzed. The case selection strategy to establish this study population was as following. Singleton pregnancies at $\geq 20$ weeks of gestation in women aged between 20 and 44 years were identified in both databases. Pregnant women with preexisting type 2 diabetes (International Classification of Diseases, Ninth Revision, Clinical Modification [ICD-9-CM] codes: 250. $\times 0,250 . \times 2$ ) from outpatient and inpatient claims were identified. Women with a diagnosis of type 1 diabetes (ICD-9-CM codes: $250 . \times 1,250 \times 3$ ) before pregnancy was excluded. Medications prescribed were identified using National Drug Codes in the NHI dataset. Three treatment groups were included in this study: 1) received insulin (insulin human, insulin lispro, insulin aspart, insulin glulisine, insulin detemir, or insulin glargine) and no oral antidiabetic drugs (metformin, sulfonylureas, dipeptidyl peptidase 4 inhibitor, thiazolidinediones, insulin secretagogues, or $\alpha$-glucosidase inhibitors) before and after becoming pregnant (insulin group); 2) received metformin but no insulin before pregnancy and switched to insulin after becoming pregnant (switching group); and 3) received metformin but no insulin before and after becoming pregnant (metformin group) (Fig. 1). Oral hypoglycemic agents (e.g., sulfonylureas) were allowed to be administered concomitantly with metformin.

\section{Covariates}

Covariates that could potentially affect pregnancy outcomes, including patient age, duration of type 2 diabetes, comorbidities (hypertension, hyperlipidemia, retinopathy) and aspirin use were identified and included in our analysis (see Supplemental Table S1). The proportion of subjects who used lipid-lowering agents (statins, 


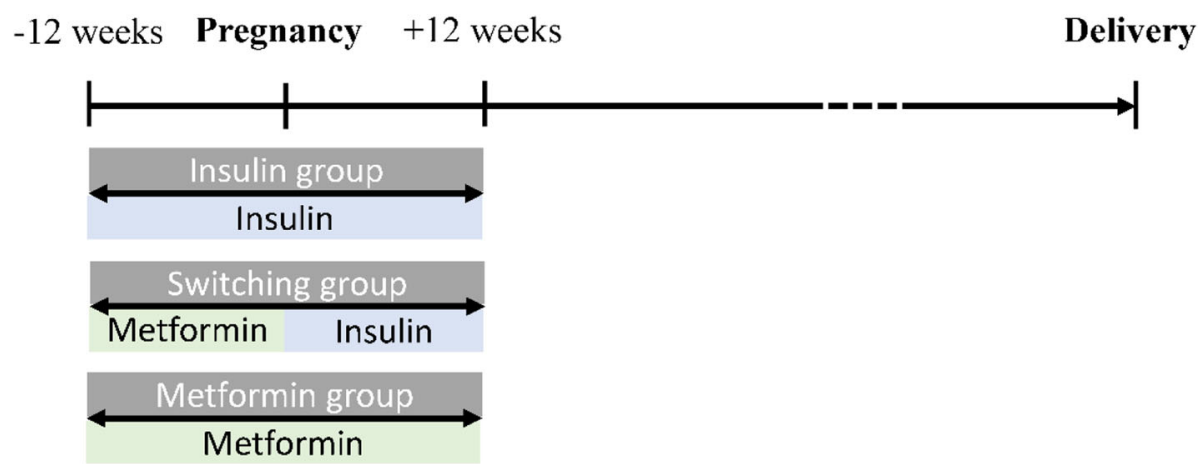

Fig. 1 Three study groups according to glucose-lowering regimens

ezetimibe or fibrates) and antihypertensive agents (angiotensin-converting-enzyme inhibitors, angiotensin receptor blockers, $\beta$-blockers, calcium channel blockers, thiazide diuretics, loop diuretics or potassium-sparing diuretics) was determined (see Supplemental Table S2). Our statistical analysis did not adjust for lipid-lowering agents and antihypertensive agents because dyslipidemia and hypertension were included as the covariates. Aspirin was included as one of the covariates because aspirin use in pregnancy has been shown to reduce the risk of preeclampsia [21]. Nephropathy and neuropathy were not included as covariates because of limited events.

\section{Study outcomes}

Adverse pregnancy outcomes of the mother and fetus, including primary cesarean section, pregnancy-related hypertension, preeclampsia, preterm birth ( $<37$ weeks), very preterm birth $(<32$ weeks), low birth weight $(<$ $2500 \mathrm{~g}$ ), high birth weight (>4000 g), small for gestational age, large for gestational age, congenital malformations, Apgar score $<7$ at $5 \mathrm{~min}$ and stillbirth were identified in the NHI and Birth Registry datasets.

\section{Statistical analyses}

Patient characteristics were analyzed to determine the mean and standard deviation (SD), the median and interquartile range (IQR) for continuous variables, and the frequency for dichotomous variables. The crude incidence rates of adverse pregnancy events were calculated using the number of events divided by the total number of pregnancies for each group. Information for events involving fewer than 5 cases was not available because of the data-use policy for the NHI dataset.

Our primary analysis targeted women who had preexisting type 2 diabetes and were eligible for this study. We conducted two subgroup analyses, one limited to the primary cohort who had had type 2 diabetes for less than 3 years and the other limited to metformin users who received metformin as monotherapy. The risks of adverse pregnancy outcomes in the switching group and the metformin group were compared with the insulin group as the reference. The adjusted odds ratios (aOR) and $95 \%$ confidence intervals $(\mathrm{CI})$ were computed using generalized estimation equations for each of the pregnancy outcomes adjusted for covariates using SAS (version 9.4; SAS Institute, Inc). The level of statistical significance in this study was set at $p<0.05$.

\section{Results}

\section{Maternal characteristics}

There were 1166 pregnancies included for the primary analysis based on case selection strategy, 222 were in the insulin group, 318 were in the switching group and 626 were in the metformin group. The baseline patient characteristics of these three treatment groups are shown in Table 1.

\section{Pregnancy outcomes}

The incidence of adverse pregnancy outcomes in each group is presented in Table 2. Using the insulin group as the reference, the risks of adverse pregnancy outcome in the switching group and the metformin group were computed using generalized estimation equations, adjusting for patient age, duration of type 2 diabetes, hypertension, hyperlipidemia, retinopathy, and aspirin use (Table 3). Compared with the insulin group, the switching group had a similar risk of primary cesarean section, pregnancy-related hypertension, preeclampsia, preterm birth, very preterm birth, low birth weight, high birth weight, large for gestational age, and congenital malformations. The metformin group had a lower risk of primary cesarean section (aOR, 0.57; 95\% CI, 0.40-0.82; $p=0.002)$ and congenital malformations (aOR, 0.51; $95 \%$ CI, $0.27-0.94 ; p=0.032$ ) compared with the insulin group. The risks of other outcomes, including pregnancy-related hypertension, preeclampsia, preterm birth, very preterm birth, low birth weight, high birth weight, small for gestational age, large for gestational 
Table 1 Study cohort characteristics

\begin{tabular}{|c|c|c|c|}
\hline & $\begin{array}{l}\text { Insulin } \\
\text { Group } \\
(n=222)\end{array}$ & $\begin{array}{l}\text { Switching } \\
\text { Group } \\
(n=318)\end{array}$ & $\begin{array}{l}\text { Metformin } \\
\text { Group } \\
(n=626)\end{array}$ \\
\hline Age at pregnancy, y, mean (SD) & $34.48(4.02)$ & $34.05(4.23)$ & $33.51(4.31)$ \\
\hline \multicolumn{4}{|l|}{ Age at pregnancy, y, No. (\%) } \\
\hline $20-29$ & 29 (13.06) & $48(15.09)$ & $129(20.61)$ \\
\hline $30-34$ & 86 (38.74) & $124(38.99)$ & $258(41.21)$ \\
\hline $35-39$ & $90(40.54)$ & $123(38.68)$ & 189 (30.19) \\
\hline $40-44$ & $17(7.66)$ & $23(7.23)$ & $50(7.99)$ \\
\hline Duration of diabetes, y, median (IQR) & $3.65(4.58)$ & $3.10(3.84)$ & $1.49(3.34)$ \\
\hline \multicolumn{4}{|l|}{ Baseline comorbidities, No. (\%) } \\
\hline Hypertension & $46(20.72)$ & $84(26.42)$ & $114(18.21)$ \\
\hline Hyperlipidemia & $110(49.55)$ & $157(49.37)$ & $145(23.16)$ \\
\hline Retinopathy & $24(10.81)$ & $21(6.60)$ & $26(4.15)$ \\
\hline Nephropathy & $<5(<2.25)^{a}$ & $<5(<1.57)^{\mathrm{a}}$ & $8(1.28)$ \\
\hline Neuropathy & $5(2.25)$ & $<5(<2.25)^{\mathrm{a}}$ & $0(0)$ \\
\hline \multicolumn{4}{|l|}{ Baseline medications, No. (\%) } \\
\hline Aspirin & $5(2.25)$ & $9(2.83)$ & $48(7.67)$ \\
\hline Statin & $17(7.66)$ & $50(15.72)$ & $38(6.07)$ \\
\hline Ezetimibe & $<5(<2.25)^{a}$ & $<5(<1.57)^{\mathrm{a}}$ & $0(0)$ \\
\hline Fibrate & $0(0)$ & $0(0)$ & $0(0)$ \\
\hline ACEI/ARB & $7(3.15)$ & $18(5.66)$ & $26(4.15)$ \\
\hline Beta-blockers & $<5(<2.25)^{\mathrm{a}}$ & $10(3.14)$ & 19 (3.04) \\
\hline Calcium channel blockers & $9(4.05)$ & $27(8.49)$ & $24(3.83)$ \\
\hline Diuretics ${ }^{b}$ & $<5(<2.25)^{\mathrm{a}}$ & $<5(<1.57)^{\mathrm{a}}$ & $12(1.92)$ \\
\hline
\end{tabular}

$S D$ standard deviation, IQR interquartile range, $A C E I$ angiotensin-converting-enzyme inhibitor, $A R B$ angiotensin II receptor blocker

${ }^{a}$ According to the data-use policy, information for events involving $<5$ cases was not available

${ }^{\mathrm{b}}$ Including thiazide diuretics, loop diuretics and potassium-sparing diuretics

age, Apgar score $<7$ at $5 \mathrm{~min}$, and stillbirth were all similar in both the metformin group and the insulin group.

\section{Additional analyses}

Two secondary analyses were performed. First, we accessed the risk of study outcomes in women with type 2 diabetes of less than 3 years duration (Table 4). There were 154 patients $(48.4 \%)$ in the switching group and 427 patients $(62.6 \%)$ in the metformin group who had had known type 2 diabetes for $<3$ years. Compared with the insulin group, the switching group had similar pregnancy-related risks for the mother and the fetus. The metformin group had lower risks of primary cesarean section (aOR, 0.50; 95\% CI, 0.30-0.83; $p=$ 0.008 ) and congenital malformations (aOR, 0.40; 95\% CI, $0.17-0.93 ; p=0.033$ ) than did the insulin group. Comparable risk in the other pregnancy outcomes was observed in both the metformin group and the insulin group. These data are consistent with the primary findings.
To address the effects of metformin versus insulin treatment on pregnancy outcomes, we limited metformin users in the switching group and metformin group to those who received metformin as monotherapy. There were 273 patients $(85.8 \%)$ in the switching group and 596 patients $(95.2 \%)$ in the metformin group who received metformin as monotherapy (Table 5). In this analysis, the switching group had a similar risk of adverse pregnancy outcomes when compared with the insulin group. The metformin group had lower risks of primary cesarean section (aOR, 0.57; 95\% CI, 0.40-0.82; $p=$ 0.003 ) and congenital malformations (aOR, 0.47; 95\% CI, $0.25-0.89 ; p=0.021)$ compared with the insulin group. The risks of the other pregnancy outcomes were similar between the metformin group and the insulin group. The results of this analysis are also consistent with the primary findings.

\section{Discussion}

This investigation is the first nationwide populationbased cohort study to assess the effects of different 
Table 2 Incidence of adverse pregnancy outcomes among women who received insulin, metformin to insulin switching or metformin therapy

\begin{tabular}{llll}
\hline Cohort & $\begin{array}{l}\text { Insulin } \\
\text { Group } \\
(n=222)\end{array}$ & $\begin{array}{l}\text { Switching } \\
\text { Group } \\
(n=318)\end{array}$ & $\begin{array}{l}\text { Metformin } \\
\text { Group } \\
(n=626)\end{array}$ \\
\hline Maternal outcome, (\%) & 38.74 & 41.19 & 23.16 \\
$\quad$ Primary cesarean section & 24.77 & 29.87 & 20.77 \\
Pregnancy-related hypertension & 16.67 & 20.13 & 13.26 \\
Preeclampsia & & & \\
Fetal outcome, (\%) & 27.93 & 31.45 & 23.16 \\
Preterm birth (< 37 week) & 3.50 & 3.77 & 3.99 \\
Very preterm birth $(<32$ weeks) & 4.50 .36 & 12.89 & 10.38 \\
Low birth weight $(<2500 \mathrm{~g})$ & 10.36 & 10.54 \\
High birth weight $(>4000 \mathrm{~g})$ & 13.96 & 19.18 & 6.23 \\
Small for gestational age & 6.76 & 5.66 & 34.82 \\
Large for gestational age & 40.54 & 49.06 & 5.27 \\
Congenital malformations & 9.91 & 7.86 & 1.81 \\
APGAR score $<7$ at 5 min & $<2.25^{\mathrm{a}}$ & 2.57 & 2.72 \\
\hline Stillbirth & 3.60 & 2.20 & \\
\hline
\end{tabular}

${ }^{a}$ According to the data-use policy, information for events involving $<5$ cases was not available

glucose-lowering regimens in women with preexisting type 2 diabetes on becoming pregnant. Our data demonstrate that metformin treatment is not associated with an increased risk of adverse pregnancy outcomes as compared with standard insulin treatment, whether continuing with metformin therapy or switching from metformin to insulin.
Our results show that many women with preexisting type 2 diabetes received metformin treatment during pregnancy. The popularity of metformin use during pregnancy in this study might result from the ease of use of this oral agent, the emergence of promising safety profiles for metformin use in pregnancy, and needle phobia [4]. The rising cost of insulin is one of the reasons supporting the use of metformin as an alternative to insulin during pregnancy [22-26]. However, NHI provides reimbursements for insulin and insulin is inexpensive in Taiwan [27]; therefore, affordability may not account for the frequent use of metformin in this study.

Our data reveal that metformin treatment did not increase the risk of adverse pregnancy outcomes. The safety of metformin use during pregnancy has been demonstrated in patients with GDM and with PCOS. In the largest study of women with GDM $(n=751)$ randomized to metformin or insulin treatment at 20-33 weeks of gestation, comparable pregnancy outcomes were observed between the two arms [12]; a follow-up study of their offspring reported that metformin-exposed children in the New Zealand cohort were larger at 7-9 years of age, although no such difference was seen in the Adelaide cohort [28]. In women with PCOS randomized to metformin or placebo treatment from the first trimester through to delivery, metformin therapy did not increase pregnancy complications over those seen with placebo [29]. However, metformin-exposed children were reported to have an increased prevalence of overweight or obesity at 4 years of age [30]. Interpretation of these follow-up data is difficult, given that postnatal

Table 3 Adjusted odds ratio of adverse pregnancy outcomes among women who received metformin to insulin switching therapy or metformin therapy as compared to insulin therapy

\begin{tabular}{|c|c|c|}
\hline Cohort & $\begin{array}{l}\text { Switching Group } \\
(n=318)^{\mathrm{a}}\end{array}$ & $\begin{array}{l}\text { Metformin Group } \\
(n=626)^{\mathrm{a}}\end{array}$ \\
\hline \multicolumn{3}{|l|}{ Maternal outcome } \\
\hline Primary cesarean section & $1.14(0.80-1.63)$ & $0.57(0.40-0.82)$ \\
\hline Pregnancy-related hypertension & $1.26(0.83-1.92)$ & $0.92(0.61-1.40)$ \\
\hline Preeclampsia & $1.24(0.75-2.05)$ & $0.92(0.57-1.49)$ \\
\hline \multicolumn{3}{|l|}{ Fetal outcome } \\
\hline Preterm birth (< 37 week) & $1.21(0.84-1.75)$ & $1.05(0.69-1.59)$ \\
\hline Very preterm delivery (<32 weeks) & $0.81(0.33-2.02)$ & $1.01(0.42-2.46)$ \\
\hline Low birth weight $(<2500 \mathrm{~g})$ & $1.32(0.76-2.29)$ & $1.30(0.75-2.25)$ \\
\hline High birth weight (> $4000 \mathrm{~g}$ ) & $1.47(0.92-2.36)$ & $0.91(0.54-1.53)$ \\
\hline Small for gestational age & - & $0.98(0.51-1.90)$ \\
\hline Large for gestational age & $1.39(0.96-2.02)$ & $0.99(0.69-1.42)$ \\
\hline Congenital malformations & $0.75(0.41-1.37)$ & $0.51(0.27-0.94)$ \\
\hline Apgar score $<7$ at 5 min & - & $1.55(0.31-7.67)$ \\
\hline Stillbirth & - & $0.82(0.28-2.36)$ \\
\hline
\end{tabular}

${ }^{a}$ Adjusted for age, duration of type 2 diabetes, hypertension, hyperlipidemia, retinopathy, and aspirin use 
Table 4 Adjusted odds ratio of adverse pregnancy outcomes in women with duration of type 2 diabetes $<3$ years received metformin to insulin switching therapy or metformin therapy, as compared to insulin therapy

\begin{tabular}{|c|c|c|}
\hline Cohort & $\begin{array}{l}\text { Switching Group } \\
(n=154)^{\mathrm{a}}\end{array}$ & $\begin{array}{l}\text { Metformin Group } \\
(n=427)^{\mathrm{a}}\end{array}$ \\
\hline \multicolumn{3}{|l|}{ Maternal outcome } \\
\hline Primary cesarean section & $1.01(0.59-1.74)$ & $0.50(0.30-0.83)$ \\
\hline Pregnancy-related hypertension & $1.65(0.86-3.16)$ & $1.08(0.55-2.11)$ \\
\hline Preeclampsia & $1.39(0.67-2.88)$ & $0.97(0.44-2.14)$ \\
\hline \multicolumn{3}{|l|}{ Fetal outcome } \\
\hline Preterm birth (< 37 week) & $1.08(0.60-1.95)$ & $0.79(0.43-1.47)$ \\
\hline Very preterm delivery (<32 weeks) & - & - \\
\hline Low birth weight $(<2500 \mathrm{~g})$ & $1.26(0.49-3.22)$ & $1.27(0.52-3.09)$ \\
\hline High birth weight (> $4000 \mathrm{~g}$ ) & - & - \\
\hline Small for gestational age & - & - \\
\hline Large for gestational age & $1.43(0.83-2.49)$ & $0.79(0.47-1.33)$ \\
\hline Congenital malformations & $0.60(0.26-1.37)$ & $0.40(0.17-0.93)$ \\
\hline Apgar score $<7$ at 5 min & - & - \\
\hline Stillbirth & - & $0.23(0.06-0.92)$ \\
\hline
\end{tabular}

${ }^{a}$ Adjusted for age, duration of type 2 diabetes, hypertension, hyperlipidemia, retinopathy, and aspirin use

factors might significantly influence obesity risk in children [22].

In the current study, the metformin group had a lower risk of congenital malformations compared with the insulin group. The mechanisms accounting for this observation are unclear. It has been reported that congenital malformations are proportional to increases in hemoglobin A1c during the first 10 weeks of pregnancy [31]. It is certainly tempting to think that women being managed with metformin might have less severe diabetes than those requiring insulin therapy, but the current databases do not provide such laboratory data in our population.

Our data reveal a higher risk of primary cesarean section in the insulin group than in the metformin group. Potential factors contributing to this observation are unclear and need to be clarified; however, maternal obesity might be involved. Maternal obesity is associated with higher rates of cesarean delivery [32]. Insulin therapy commonly results in weight gain, while metformin

Table 5 Adjusted odds ratio of adverse pregnancy outcomes among women who received metformin monotherapy in switching group and metformin group as compared to insulin therapy

\begin{tabular}{|c|c|c|}
\hline Cohort & $\begin{array}{l}\text { Switching Group } \\
(n=273)^{\mathrm{a}}\end{array}$ & $\begin{array}{l}\text { Metformin Group } \\
(n=596)^{\mathrm{a}}\end{array}$ \\
\hline \multicolumn{3}{|l|}{ Maternal outcome } \\
\hline Primary cesarean section & $1.06(0.73-1.54)$ & $0.57(0.40-0.82)$ \\
\hline Pregnancy-related hypertension & $1.20(0.78-1.86)$ & $0.81(0.53-1.23)$ \\
\hline Preeclampsia & $1.17(0.70-1.95)$ & $0.83(0.51-1.34)$ \\
\hline \multicolumn{3}{|l|}{ Fetal outcome } \\
\hline Preterm birth (< 37 week) & $1.07(0.73-1.58)$ & $0.97(0.64-1.48)$ \\
\hline Very preterm delivery (<32 weeks) & $0.51(0.18-1.49)$ & $0.93(0.38-2.27)$ \\
\hline Low birth weight $(<2500 \mathrm{~g})$ & $1.14(0.64-2.04)$ & $1.33(0.76-2.34)$ \\
\hline High birth weight (> $4000 \mathrm{~g}$ ) & $1.60(0.98-2.60)$ & $0.84(0.49-1.45)$ \\
\hline Small for gestational age & - & $1.00(0.51-1.97)$ \\
\hline Large for gestational age & $1.54(1.05-2.25)$ & $0.86(0.60-1.23)$ \\
\hline Congenital malformations & $0.72(0.38-1.35)$ & $0.47(0.25-0.89)$ \\
\hline Apgar score $<7$ at 5 min & - & $1.48(0.28-7.79)$ \\
\hline Stillbirth & - & $0.79(0.26-2.39)$ \\
\hline
\end{tabular}

${ }^{a}$ Adjusted for age, duration of type 2 diabetes, hypertension, hyperlipidemia, retinopathy, and aspirin use 
treatment has weight-neutral or weight-sparing effects $[33,34]$.

We found similar pregnancy outcomes between the switching group and the insulin group. The initiation and intensification of insulin therapy are usually difficult and challenging with respect to achieving tight glycemic control and preventing hypoglycemia [35]. Our results reveal that switching treatment from metformin to insulin did not increase the risk of adverse pregnancy outcomes over that of standard insulin treatment.

In this study, we found 58 pregnancies were treated with glyburide, another alternative oral glucose-lowering agent for pregnant women with type 2 diabetes. The limited number of glyburide users prevented further statistical analyses.

The present study has several strengths. First, the patient cohort was drawn from a nationwide database with a relatively large number of pregnant women with type 2 diabetes compared with prior reports [9-13]. Second, its design included active treatment comparison of clinically relevant drugs, including insulin and metformin. Both drugs are recommended for the treatment of pregnant women with type 2 diabetes. Third, the results are consistent across analyses of the primary cohort and two subgroups, supporting the validity of these findings. Fourth, our findings are in line with prior reports addressing the safety of metformin use in pregnancy [9-14].

This study has some limitations. First, pregnant women with type 2 diabetes treated with insulin may have poor glycemic control and a higher rate of comorbidities at the time of conception, which may lead to adverse pregnancy outcomes. We used surrogate indicators, including duration of type 2 diabetes and retinopathy, to adjust for the diabetes severity and also adjusted for comorbidities, including hypertension and hyperlipidemia. Second, this study did not assess pregnancy outcomes before 20 weeks of gestation because of the limit of the Taiwan Birth Registry dataset. However, a randomized placebo-controlled trial has reported that the use of metformin improves live-birth rates in women with PCOS [11]. Third, long-term outcomes for offspring were not evaluated in this study. Further studies to address this issue are mandatory. Fourth, the diagnoses of pregnancy-related hypertension and preeclampsia were based on ICD-9-CM codes, rather than based on clinical characteristics of individual patient in this study. Using ICD-9-CM codes may carry the risks of misclassification and underrepresentation. Of note, preeclampsia was diagnosed in $15.8 \%$ of pregnancies in this study, which is similar to prior reports showing $10-14 \%$ women with type 2 diabetes had preeclampsia [36, 37]. Fifth, some covariates, such as glycemic control, prepregnancy body mass index and gestational weight gain were not included in our adjusted analyses because of the limited availability of NHI datasets. Sixth, propensity score matching was not performed to confirm our primary findings because of the relatively small sample size of each treatment group.

\section{Conclusions}

In conclusion, our data reveal that metformin treatment for women with type 2 diabetes in pregnancy is not associated with an increased risk of adverse pregnancy outcomes compared with standard insulin therapy.

\section{Supplementary information}

Supplementary information accompanies this paper at https://doi.org/10. 1186/s12884-020-03207-0.

Additional file 1: Supplemental Table S1. Diseases and outcomes.

Supplemental Table S2. List of medications.

\begin{abstract}
Abbreviations
PCOS: Polycystic Ovary Syndrome; GDM: Gestational Diabetes Mellitus; NHI: National Health Insurance; ICD-9-CM: International Classification of Diseases, Ninth Revision, Clinical Modification; SD: Standard Deviation; IQR: Interquartile Range; aOR: adjusted Odds Ratio; Cl: Confidence Interval
\end{abstract}

\section{Acknowledgements}

We thank the statistical assistance and wish to acknowledge the support of the Maintenance Project of the Center for Big Data Analytics and Statistics (Grant CLRPG3D0046) at Chang Gung Memorial Hospital for study design and monitor, data analysis and interpretation. We also thank the Health and Welfare Data Science Center from the Ministry of Health and Welfare in Taiwan for provided the database for this study.

\section{Authors' contributions}

SFL and YTH conceived of and designed the study. SHC and CFK were in charge of the data collection. WTL and MJC provided the analysis of the data, statistical analyses and made the tables and figures. SHL and YTH drafted the manuscript, and critical discussions involved SHC, CFK, WTL, and MJC. YTH takes responsibility for the study as a whole, and all authors read and approved the final manuscript.

\section{Funding}

This study was funded (CORPG3J0031) by Chang Gung Memorial Hospital, Linkou, Taiwan. But, the funder had no any other role in this study.

\section{Availability of data and materials}

To secure the participants' privacy, NHI research database and Taiwan Birth Registry data (the datasets of this study used) cannot be accessed outside the Health and Welfare Data Science Center, Ministry of Health and Welfare, Taiwan. The researchers must analyze these datasets at the Health and Welfare Data Science Center only.

\section{Ethics approval and consent to participate}

This retrospective cohort study was approved by the Institutional Review Board (IRB) of Chang Gung Memorial Hospital in Taiwan (IRB number: 201800556B0) and was conducted in accordance with the ethical principles. The IRB waived the requirement of patient informed consent due to the anonymized nature of these datasets.

Consent for publication

Not applicable.

Competing interests

The authors declare that they have no competing interests. 


\section{Author details}

'Division of Endocrinology and Metabolism, Department of Internal Medicine, New Taipei Municipal TuCheng Hospital, New Taipei City, Taiwan. ${ }^{2}$ Division of Endocrinology and Metabolism, Department of Internal Medicine, Chang Gung Memorial Hospital, Taoyuan, Taiwan. ${ }^{3}$ College of Medicine, Chang Gung University, Taoyuan, Taiwan. ${ }^{4}$ Division of Cardiology, Department of Internal Medicine, Chang Gung Memorial Hospital, Taoyuan, Taiwan. ${ }^{5}$ Center for Big Data Analytics and Statistics, Chang Gung Memorial Hospital, Linkou, No.15, Wunhua 1st Rd., Gueishan Dist, Taoyuan City 333, Taiwan. ${ }^{6}$ Graduate Institute of Nursing, Chang Gung University of Science and Technology, Taoyuan, Taiwan. 'Division of Rheumatology, Allergy and Immunology, Department of Internal Medicine, Chang Gung Memorial Hospital, Taoyuan, Taiwan. ${ }^{8}$ Center for Artificial Intelligence in Medicine, Chang Gung Memorial Hospital, Taoyuan, Taiwan.

Received: 3 February 2020 Accepted: 25 August 2020

Published online: 04 September 2020

\section{References}

1. Temple R, Murphy H. Type 2 diabetes in pregnancy - an increasing problem. Best Pract Res Clin Endocrinol Metab. 2010;24:591-603.

2. Roland JM, Murphy HR, Ball V, et al. The pregnancies of women with type 2 diabetes: poor outcomes but opportunities for improvement. Diabet Med. 2005;22:1774-7.

3. American Diabetes Association. Management of diabetes in pregnancy: standards of medical care in diabetes-2019. Diabetes Care. 2019;42:S165-72.

4. Polasek TM, Doogue MP, Thynne TRJ. Metformin treatment of type 2 diabetes mellitus in pregnancy: update on safety and efficacy. Ther Adv Drug Saf. 2018;9:287-95.

5. Karter AJ, Subramanian U, Saha C, et al. Barriers to insulin initiation: the translating research into action for diabetes insulin starts project. Diabetes Care. 2010;33:733-5.

6. UK Prospective Diabetes Study (UKPDS) Group. Effect of intensive bloodglucose control with metformin on complications in overweight patients with type 2 diabetes (UKPDS 34). Lancet. 1998;352:854-65.

7. Holman RR, Paul SK, Bethel MA, et al. 10-year follow-up of intensive glucose control in type 2 diabetes. N Engl J Med. 2008:359:1577-89.

8. American Diabetes Association. Pharmacologic approaches to glycemic treatment: standards of medical care in diabetes_-2019. Diabetes Care. 2019;42:S90-102.

9. Jakubowicz DJ, luorno MJ, Jakubowicz $S$, et al. Effects of metformin on early pregnancy loss in the polycystic ovary syndrome. J Clin Endocrinol Metab. 2002;87:524-9.

10. Bolton S, Cleary B, Walsh J, et al. Continuation of metformin in the first trimester of women with polycystic ovarian syndrome is not associated with increased perinatal morbidity. Eur J Pediatr. 2009:168:203-6.

11. Morin-Papunen $L$, Rantala AS, Unkila-Kallio L, et al. Metformin improves pregnancy and live-birth rates in women with polycystic ovary syndrome (PCOS): a multicenter, double-blind, placebo-controlled randomized trial. J Clin Endocrinol Metab. 2012:97:1492-500.

12. Rowan JA, Hague WM, Gao W, et al. Metformin versus insulin for the treatment of gestational diabetes. N Engl J Med. 2008;358:2003-15.

13. Chiswick C, Reynolds RM, Denison F, et al. Effect of metformin on materna and fetal outcomes in obese pregnant women (EMPOWaR): a randomised, double-blind, placebo-controlled trial. Lancet Diabetes Endocrino. 2015;13: 778-86

14. Given JE, Loane M, Garne E, et al. Metformin exposure in first trimester of pregnancy and risk of all or specific congenital anomalies: exploratory casecontrol study. BMJ. 2018;361:k2477.

15. Charles B, Norris R, Xiao X, et al. Population pharmacokinetics of metformin in late pregnancy. Ther Drug Monit. 2006;28:67-72.

16. Lindsay RS, Loeken MR. Metformin use in pregnancy: promises and uncertainties. Diabetologia. 2017;60:1612-9.

17. The National Diabetes Services Scheme (NDSS; administered by Diabetes Australia). Having a healthy baby guide for women with type 2 diabetes. Available: https://www.ndss.com.au/about-diabetes/resources/find-aresource/having-a-healthy-baby-guide-for-women-with-type-2-diabetes. Accessed 2020 April 11.

18. Hsing AW, loannidis JP. Nationwide population science: lessons from the Taiwan National Health Insurance research database. JAMA Intern Med. 2015;175:1527-9.
19. Chang SH, Chou IJ, Yeh YH, et al. Association between use of non-vitamin K oral anticoagulants with and without concurrent medications and risk of major bleeding in nonvalvular atrial fibrillation. JAMA. 2017;318:1250-9.

20. Lin CM, Lee PC, Teng SW, et al. Validation of the Taiwan birth registry using obstetric records. J Formos Med Assoc. 2004;103:297-301.

21. Atallah A, Lecarpentier $E$, Goffinet $F$, et al. Aspirin for prevention of preeclampsia. Drugs. 2017;77:1819-31.

22. Barbour LA, Feig DS. Metformin for gestational diabetes mellitus: progeny, perspective, and a personalized approach. Diabetes Care. 2019;42:396-9.

23. Society of Maternal-Fetal Medicine (SMFM) Publications Committee. SMFM statement: pharmacological treatment of gestational diabetes. Am J Obstet Gynecol. 2018;218:B2-4.

24. National Institute for Health and Care Excellence (NICE). Diabetes in pregnancy: management from preconception to the postnatal period. NICE guideline NG3. Available from https://www.nice.org.uk/guidance/ng3. Accessed 19 March 2019

25. Hod M, Kapur A, Sacks DA, et al. The International Federation of Gynecology and Obstetrics (FIGO) initiative on gestational diabetes mellitus: a pragmatic guide for diagnosis, management, and care. Int J Gynaecol Obstet. 2015; 131(Suppl 3):S173-211.

26. Feig DS, Berger H, Donovan L, et al. Diabetes and pregnancy. Can J Diabetes. 2018:42(Suppl 1):S255-82.

27. Sable-Smith B. Americans going to Mexico to buy insulin at fraction of US cost. Medscape Feb 15, 2019. Available from https://www.medscape.com/ viewarticle/909174. Accessed 19 March 2019.

28. Rowan JA, Rush EC, Plank LD, et al. Metformin in gestational diabetes: the offspring follow-up (MiG TOFU): body composition and metabolic outcomes at 7-9 years of age. BMJ Open Diabetes Res Care. 2018;6:e000456.

29. Vanky E, Stridsklev S, Heimstad R, et al. Metformin versus placebo from first trimester to delivery in polycystic ovary syndrome: a randomized, controlled multicenter study. J Clin Endocrinol Metab. 2010;95:E448-55.

30. Engen Hanem LG, Stridsklev S, Júlíusson PB, et al. Metformin use in PCOS pregnancies increases the risk of offspring overweight at 4 years of age: follow-up of two RCTs. J Clin Endocrinol Metab. 2018;103:1612-21.

31. Guerin A, Nisenbaum R, Ray JG. Use of maternal GHb concentration to estimate the risk of congenital anomalies in the offspring of women with prepregnancy diabetes. Diabetes Care. 2007;30:1920-5.

32. Sebire NJ, Jolly M, Harris JP, et al. Maternal obesity and pregnancy outcome: a study of 287,213 pregnancies in London. Int J Obes Relat Metab Disord. 2001;25:1175-82.

33. Butalia S, Gutierrez L, Lodha A, et al. Short- and long-term outcomes of metformin compared with insulin alone in pregnancy: a systematic review and meta-analysis. Diabet Med. 2017;34:27-36.

34. Golay A. Metformin and body weight. Int J Obes. 2008:32:61-72.

35. Wallia A, Molitch ME. Insulin therapy for type 2 diabetes mellitus. JAMA. 2014;311:2315-25

36. Knight KM, Thornburg LL, Pressman EK. Pregnancy outcomes in type 2 diabetic patients as compared with type 1 diabetic patients and nondiabetic controls. J Reprod Med. 2012;57:397-404.

37. Groen B, Links TP, van den Berg PP, et al. Similar adverse pregnancy outcome in native and nonnative dutch women with pregestational type 2 diabetes: a multicentre retrospective study. ISRN Obstet Gynecol. 2013;2013: 361435 .

\section{Publisher's Note}

Springer Nature remains neutral with regard to jurisdictional claims in published maps and institutional affiliations.

\section{Ready to submit your research? Choose BMC and benefit from:}

- fast, convenient online submission

- thorough peer review by experienced researchers in your field

- rapid publication on acceptance

- support for research data, including large and complex data types

- gold Open Access which fosters wider collaboration and increased citations

- maximum visibility for your research: over $100 \mathrm{M}$ website views per year

At $\mathrm{BMC}$, research is always in progress.

Learn more biomedcentral.com/submission 